\title{
Effect of Intestinal Cleaning Knowledge Training on Intestinal Cleaning Rate of Enteroscopy in Hospitalized Patients
}

\author{
Chen Zhaolin ${ }^{1}$, Li Guanhong ${ }^{1}$, Liang Dongxiu ${ }^{1}$, Ye Weixiang ${ }^{1}$, Luo Jinglan ${ }^{2}$, , Wei Hongcheng ${ }^{1}$, * \\ ${ }^{1}$ Department of Gastroenterology, First Affiliated Hospital of Jinan University, Guangzhou, China \\ ${ }^{2}$ Department of Internal Medicine, First Affiliated Hospital of Jinan University, Guangzhou, China
}

Email address:

tIjI@jnu.edu.cn (Luo Jinglan), twhch@jnu.edu.cn (Wei Hongcheng)

${ }^{*}$ Corresponding author

To cite this article:

Chen Zhaolin, Li Guanhong, Liang Dongxiu, Ye Weixiang, Luo Jinglan, Wei Hongcheng. Effect of Intestinal Cleaning Knowledge Training on Intestinal Cleaning Rate of Enteroscopy in Hospitalized Patients. American Journal of Nursing Science. Vol. 9, No. 3, 2020 , pp. 127-131. doi: 10.11648/j.ajns.20200903.19

Received: February 29, 2020; Accepted: April 15, 2020; Published: May 14, 2020

\begin{abstract}
Objective To investigate the clinical effectiveness of intestinal cleaning knowledge training on intestinal cleaning rate of inpatients undergoing colonoscopy. Methods A comparative study was conducted by selection of 1166 inpatients undergoing colonoscopy in our hospital from January to November in 2016. Among them, 510 inpatients from January to June 2016 were induced into the pre-training group and 656 inpatients from July to December 2016 were induced into in the post-training group. The training contents included the indications and contraindications of colonoscopy, the dietary guidance before colonoscopy, the usage of laxatives for colonoscopy, the standards of intestinal cleanliness, and the precautions before and after colonoscopy. The effective intestinal cleansing rates of the two groups were observed and recorded. Results The average score of intestinal cleaning knowledge assessment of nurses increased from 83.5 before the training to 93.1 after the training; the effective intestinal cleansing rate from July to September of the same year was continuously improved by the training combined rectification, increasing from $73.1 \%$ to $92.3 \%$, which was above the target value of $91.2 \%$. Conclusion Intestinal cleaning knowledge training plays a significant role in improvement of the rate of effective intestinal cleaning, which can significantly reduce the rate of missed diagnosis and misdiagnosis.
\end{abstract}

Keywords: Knowledge Training, Inpatients, Colonoscopy, Intestinal Cleanliness Rate

\section{Introduction}

Colonoscopy is one of the common methods to diagnose intestinal diseases, and intestinal cleanliness is an important factor to improve the success rate of colonoscopy, which plays an important role in avoidingthe missed diagnosis and misdiagnosis $[1,2]$. During intestinal preparation, inpatients are under the guidance of professional medical staff, who can timely observe the progress of intestinal cleanliness preparation of inpatients, and guide them at any time to give them great help and security [3, 4]. This study was conducted to understand the influence of intestinal cleanliness of inpatients and outpatients on the improvement of positive rate of diagnosis, thereby improving the quality of enteroscopy and ensuring the safety of patients $[5,6]$.

\section{Data and Methods}

\subsection{General Data}

A total of 1166 inpatients who underwent colonoscopy in our hospital from January 2016 to November 2016 were selected as subjects. Inclusion criteria inculded (1) 18 to 80 years old, (2) colonoscopy is required, (3) none of mental illness. Among them, 656 cases in the post-training group received one-to-one training guidance. 510 cases in the pre-training group received general routine nursing care. In the post-training group, there were 394 males and 262 females with an average age of $(56.6 \pm 7.8)$ years and an average height of $(167 \pm 5.2) \mathrm{cm}$. In the pre-training group, there were 332 
males and 178 females with an average age of $(55.1 \pm 8.7)$ years and an average height of $(165 \pm 6.1) \mathrm{cm}$. Statistical analysis of baseline data between the two groups showed no statistically significant differences $(\mathrm{P}>.05)$, indicating comparability.

\subsection{Method}

1. Before the training

(1) the nursing staff routinely instructed the patient to undergo the intestinal preparation;

(2) questionnaire was used to collect and analyze the reasons for ineffective intestinal cleaning, including the investigation on the causes of invalid intestinal cleaning in patients and the investigation on nursing staff's knowledge of intestinal cleaning.

(3) formulated training programs based on results.

2. After the training

(1) the training contents included the indications and contraindications of colonoscopy, the dietary guidance before colonoscopy, the usage of laxatives for colonoscopy, the standards of intestinal cleanliness, and the precautions before and after colonoscopy.

(2) cause analysis of nurses' incomplete grasp of intestinal cleaning knowledge;

(3) retraining nurses with incomplete knowledge: Identified specific knowledge points not mastered by nursing staff; One-on-one clinical training for nursing staff; Carried out the clinical field assessment until comprehensive grasp.

(4) co-training in nurse-patient: Make publicity materials related to intestinal cleaning, bring which to the patients and read with themwhen the nurses distribute laxatives; Handed out a reminder sheet for taking intestinal preparations.

(5) on-site guidance from nursing staff: Explained the time and method of taking laxatives to the patients, and asked the patient to repeat it; Attached a dissolution measuring cup to the laxatives and urge the patient to walk more after taking the laxatives; Patients with constipation communicated with their doctors that they took Dumick, paraffin oil or enema ahead of 3 days.

(6) develop corrective measures: checked the colonoscopy registration book every day after checking the doctor's advice in P1 and P2 class, and maked sure there was no missed registration; Maked a sign card for colonoscopy, and when the nurse issued laxatives at the bedside for propaganda, inserted the sign card above the bedside card. Director announced in the morning meeting: all patients' laxatives distribution were carried out by nurses, which could not only reduce the workload of doctors but also to avoid missed registration and ensure the quality of the mission; Explained the procedure and importance of the examination to the patients during the mission.

\subsection{Evaluation Criteria}

Grade I: A good intestinal preparation, no feces or a small amount of clear liquid, a clear vision, no impact on the operation.

Grade II: A little good intestinal preparation, a small amount of feces or more clear liquid, a clear vision on the whole, no impact on the operation basically.

Grade III: A poor Intestinal preparation, more fecesor more turbid fluid with feces, a blurred vision, a impact on the operation as shown in figure 1 .

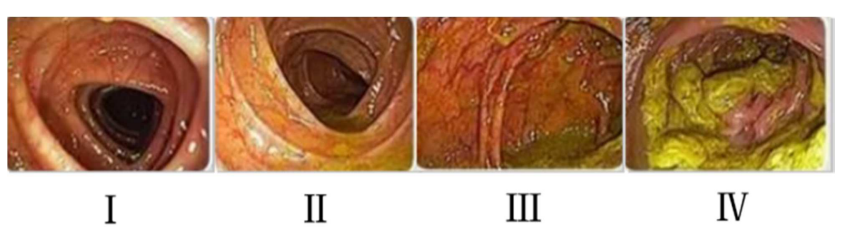

Figure 1. Evaluation criteria for intestinal cleanliness under colonoscopy.

Therefore, the target value is that the non-qualified rate of intestinal cleaning for colonoscopy in inpatients was reduced from the current $26.9 \%$ to $8.8 \%$ before December $31,2016$. That was, the qualified rate of intestinal cleaning reached $91.2 \%$ or more.

\subsection{Statistical Methods}

1. Calculation method of effective intestinal cleansing rate:

$$
\text { Effective intestinal cleansing rate }=\frac{\text { Effective intestinal cleansing cases }}{\text { Actual number of colonoscopy cases }} \times 100 \%
$$

Note: Grade I and grade II are effective intestinal cleaning. Grade III is invalid intestinal cleansing

2. Accounting of the project target value (using the improved capacity estimation method)

Target value $=$ current situation value $-($ current situation value $\times$ key improvement $\times$ team member ability $)=$ $26.9 \%(26.9 \% \times 0.84 \times 80 \%)=8.8 \%$

The capacity of team members was $80 \%$ : considering that the whole process was dominated by doctors instead of nurses, and there might be objective reasons in the process of intestinal cleaning that the nurses could not solve, ultimately, the rate was set as $80 \%$.

\section{Results}

\subsection{Cause Analysis of Patients with Ineffective Intestinal Cleaning Before Training}

Before the training, the ineffective intestinal cleaning of 
patients was analyzed, and the improper factors were as follows: wrong method of taking laxatives accounted for $35 \%$, a history of constipation accounted for $31 \%$, wrong time of taking laxatives accounted for $26 \%$, a bed rest after taking laxatives accounted for $23 \%$, and aerror diet before colonoscopy accounted for $22 \%$, as shown in figure 2 .

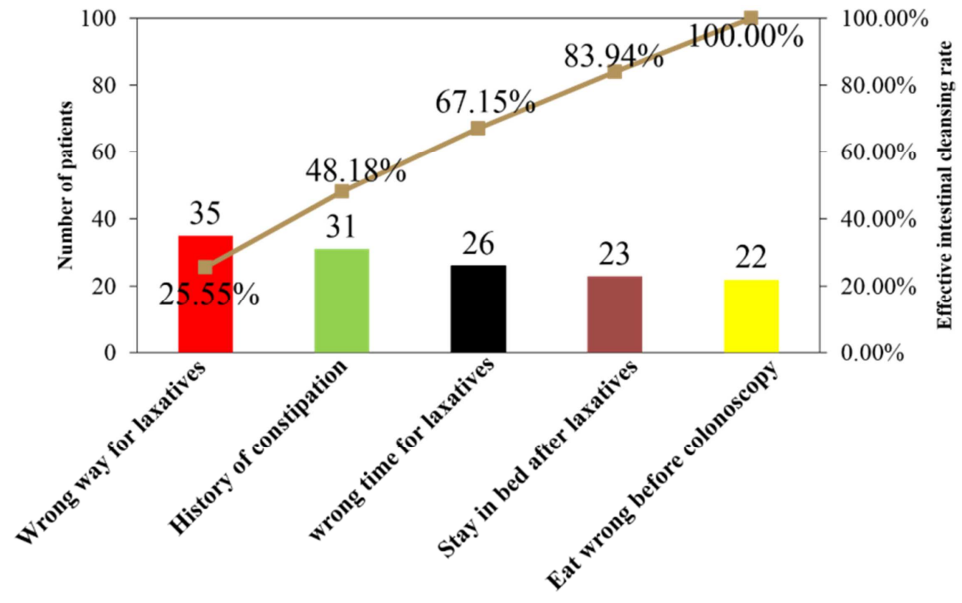

Figure 2. Ineffective attribution analysis of 137 cases of intestinal cleaning.

\subsection{Investigation on Nurses' Knowledge of Intestinal Cleaning}

The knowledge of intestinal cleaning of nursing staff was analyzed, and it found that the following situations awareness rates of nursing staff was as follows:: $44.5 \%$ of nurses were followed up the patients' defecation after patients took laxatives, $50 \%$ had an emergency management after failure of taking laxatives, $61.1 \%$ knew the correct time and method of taking laxatives, $77.8 \%$ knew the precautions for intestinal cleaning, and 83.3\%imparted dietary guidance before intestinal cleaning, as shown in table 1.

\subsection{Analysis of Nursing Staff's Mastery of Intestinal Cleaning Knowledge After Training}

The average score of intestinal cleaning knowledge assessment of nurses was from 83.5 before the training to 93.1 after the training, as shown in figure 3.

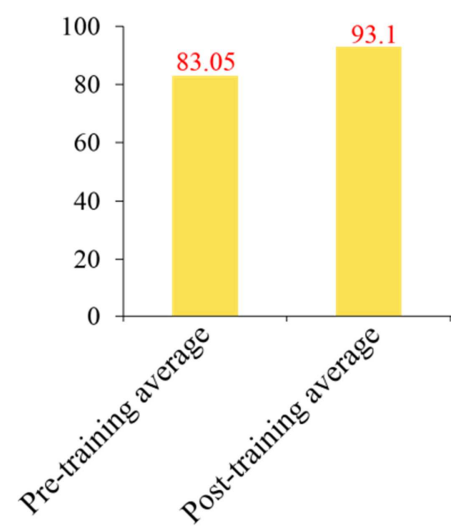

Figure 3. Comparison of assessment scores of nurses before and after training.

\subsection{Analysis of Effective Intestinal Cleaning Rate After Training}

After the training, the effective intestinal cleanliness rate from July to November 2016 was $87.6 \%$ in July, $91.3 \%$ in August, $92 \%$ in September, $94.3 \%$ in October and $96.1 \%$ in November. Before the training, the effective intestinal cleaning rate was $73.1 \%$, and after the training, the overall effective intestinal cleaning rate was $92.3 \%$ whilethe target value was $91.2 \%$, as shown in figures 4 and 5 .

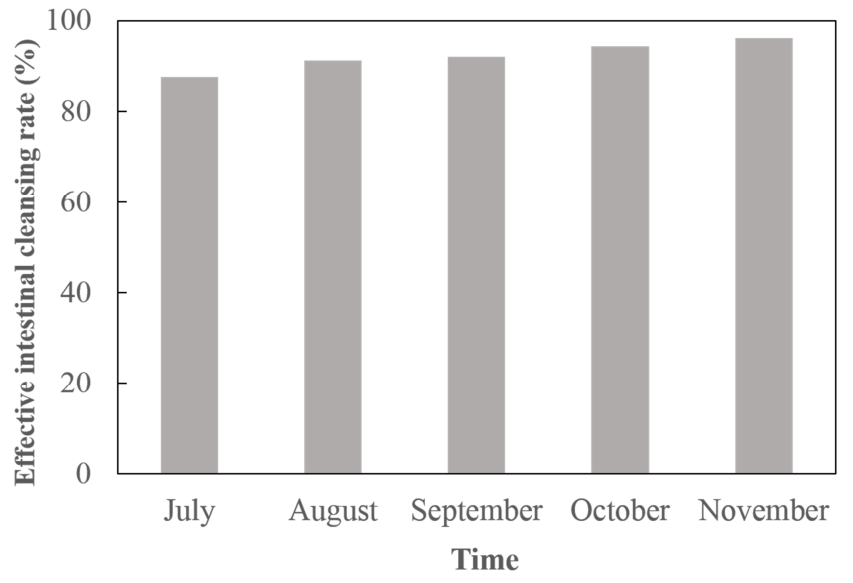

Figure 4. Effective intestinal cleansing rate after training.

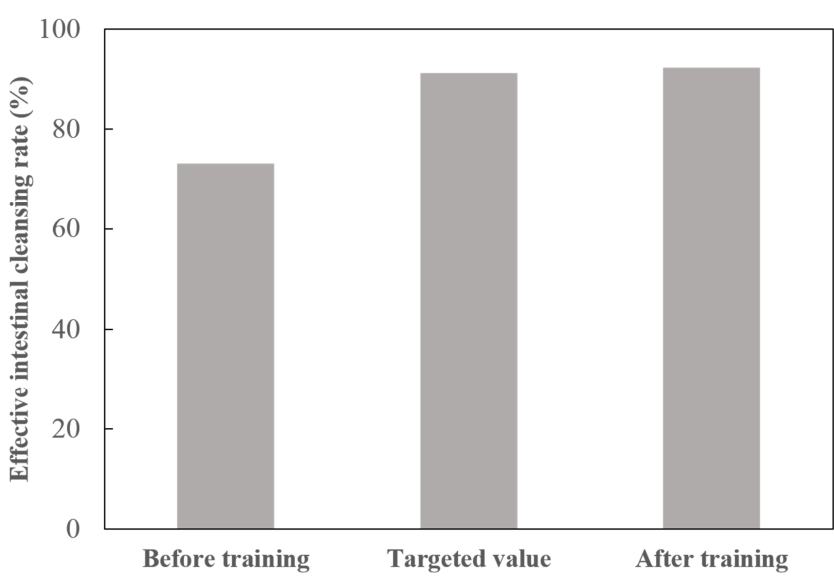

Figure 5. Effective intestinal cleansing rate before and after training. 


\section{Discussion}

1. The training has improved the knowledge of intestinal cleaning of nurses

As can be seen from table 1 and figure 3 , the training has a significant improvement on nurses' mastery of intestinal cleaning knowledge, and the average score of nurses' assessment of intestinal cleaning knowledge has increased from 83.5 before the training to 93.1 after the training [7]. Nursing staff's knowledge of intestinal cleaning has an impact on effective intestinal cleaning rate from the following aspects: (1) dietary guidance before intestinal cleaning; (2) notes on intestinal cleaning; (3) the correct time and method to take laxatives; (4) the emergency treatment after the failure of taking laxatives; (5) the patients' defecation after they take laxatives.

Table 1. Investigation and analysis of nurses' knowledge of intestinal cleaning.

\begin{tabular}{|c|c|c|c|}
\hline Project & known & unknown & Knowing rate \\
\hline Dietary guidance before intestinal cleansing & 15 & 3 & $83.3 \%$ \\
\hline Notes on intestinal cleaning & 14 & 4 & $77.8 \%$ \\
\hline The correct time and method to take laxatives & 11 & 7 & $61.1 \%$ \\
\hline Follow up after taking laxatives & 8 & 10 & $44.5 \%$ \\
\hline
\end{tabular}

$\mathrm{n}=18$, the questionnaire recovery rate was $100 \%$

2. Training combined with rectification can achieve the target value of effective intestinal cleaning

As can be seen from figure 4 and figure 5, the effective intestinal cleansing rate from July to September of that year improved continuously due to the training combined rectification, from $73.1 \%$ before the training to $92.3 \%$ after the training above the target value.

Currently, colonoscopy has played an irreplaceable role in diagnosing and treating colon diseases, and more and more people have undergoed colonoscopy to screen for colon diseases [8, 9]. Undoubtedly, intestinal preparation is a prerequisite for colonoscopy [10]. However, the rate of intestinal cleansing is still unsatisfactory because of insufficient knowledge of bowel preparation in patients, insufficient compliance, a long appointment waiting times, insensitivity to laxatives, a history of pelvic/abdominal surgery and so on [11-14]. Therefore, how to improve the rate of intestinal cleaning is still a problem worth considering. The reasons for ineffective intestinal cleaning were analyzed from the perspectives of nurses and patients, and the two factors affecting effective intestinal cleaning rate of nurses and the source of patients were respectively obtained [15]. Personalized rectification plans were formulated from the source, training was strictly implemented, and unqualified nurses were retrained and guided until they were qualified. Further, the inappropriate intestinal preparation method was corrected by the way of co-training of nurses and patients and on-site guidance for patients, so as to achieve the target value of effective intestinal cleaning [16].

\section{Conclusion}

This research adopts the training of intestinal cleaning knowledge to enhance team spirit, enhance communication and coordination ability, enhance nursing staff responsibility, improve nursing staff expertise, popularize knowledge of intestinal cleaning and lighten the patients' psychological burden, and improve the intestinal cleanliness and success rate of colonoscopy, with a great significance. It fully embodies the "people-oriented" concept of nursing, and conforms to the biological - psychology - social medical model transformation, which is well worth advocating.

\section{References}

[1] Xiao-lan W, Wei-rong L, Hua-lan Z, et al. The Effect of Bedside Handover Sheet on Nursing Care in Hepatobiliary Surgery. American Journal of Nursing. 2018; 7 (3): 84-87.

[2] Street M, Eustace P, Livingston PM, et al. Communication at the bedside to enhance patient care: A survey of nurses' experience and perspective of handover. International Journal of Nursing Practice. 2011; 17 (2): 133-140.

[3] Starmer AJ, Spector ND, West DC, et al. Integrating Research, Quality Improvement, and Medical Education for Better Handoffs and Safer Care: Disseminating, Adapting, and Implementing the I-PASS Program. Joint Commission Journal on Quality and Patient Safety. 2017; 43 (7): 319-329.

[4] Mardis T, Mardis M, Davis J, et al. Bedside Shift-to-Shift Handoffs A Systematic Review of the Literature. Journal of Nursing Care Quality. 2016; 31 (1): 54-60.

[5] Welsh CA, Flanagan ME, Ebright P. Barriers and facilitators to nursing handoffs: Recommendations for redesign. Nursing Outlook. 2010; 58 (3): 148-154.

[6] Smeulers M, Lucas C, Vermeulen H. Effectiveness of different nursing handover styles for ensuring continuity of information in hospitalised patients. Cochrane Database of Systematic Reviews. 2014 (6).

[7] Chaboyer W, Johnson J, Hardy L, et al. Transforming care strategies and nursing-sensitive patient outcomes (vol 66, pg 1111, 2010). Journal of Advanced Nursing. 2014; 70 (2): 471-471.

[8] Helsingen, L. M., et al., Colorectal cancer screening with faecal immunochemical testing, sigmoidoscopy or colonoscopy: a clinical practice guideline. BMJ, 2019. 367: p. 15515.

[9] Peterse, E., et al., The impact of the rising colorectal cancer incidence in young adults on the optimal age to start screening: Microsimulation analysis I to inform the American Cancer Society colorectal cancer screening guideline. Cancer, 2018. 124 (14): p. 2964-2973. 
[10] Shine, R., A. Bui and A. Burgess, Quality indicators in colonoscopy: an evolving paradigm. ANZ J Surg, 2020. 90 (3): p. $215-221$.

[11] Kutyla, M. J., et al., An Evidence-based Approach Towards Targeted Patient Education to Improve Bowel Preparation for Colonoscopy. J Clin Gastroenterol, 2019.

[12] Chan, W. K., et al., Appointment waiting times and education level influence the quality of bowel preparation in adult patients undergoing colonoscopy. BMC Gastroenterol, 2011. 11: p. 86 .

[13] Rotondano, G., et al., Quality of bowel cleansing in hospitalized patients undergoing colonoscopy: A multicentre prospective regional study. Dig Liver Dis, 2015. 47 (8): p. 669-74.

[14] Gandhi, K., et al., Patient Characteristics Associated With Quality of Colonoscopy Preparation: A Systematic Review and Meta-analysis. Clin Gastroenterol Hepatol, 2018. 16 (3): p. 357-369. e10.

[15] Tobiano G, Chaboyer W, McMurray A. Family members' perceptions of the nursing bedside handover. Journal of Clinical Nursing. 2013; 22 (1-2): 192-200.

[16] Chui MA, Stone JA. The prescription handoff in community pharmacy: A study of its form and function. Journal of the American Pharmacists Association. 2012; 52 (6): E161-E167. 